\title{
Körnung und Schwermineralbestand als Kriterien für eine Deckschicht in der Umgebung von Seefeld und Leutasch (Tirol)
}

\author{
Ernst Schönhals \& Thomas J. Poetsch*)
}

Cover layer, limestone gravels, grain size distribution, weathering, heavy minerals, eolian transport Tyrol

K u r z f a s s u n g : Mit Hilfe der Korngrößenverteilung und der Schwermineralanalyse wurde nachgewiesen, daß der auf Kalkschottern entwickelte Boden nicht in situ, sondcrn aus einem überwiegend äolischen Substrat (Deckschicht) entstanden ist. Es handelt sich infolgedessen um ein zweischichtiges Profil.

[Granulation and Heavy Mineral Content as Criteria for a Cover Layer in the Area of Seefeld and Leutasch (Tyrol)]

A bstract: With the help of the grain size distribution and the heavy mineral analysis it was proved that the soil lying on top of the limestone gravels dit not develop there but was formed by an eolian deposit (cover layer) instead. It is therefore a profile consisting of two layers.

\section{Einleitung}

Quartärgeologische, geomorphologische und bodenkundliche Untersuchungen der letzten beiden Jahrzehnte haben ergeben, daß während der Jüngeren Tundrenzeit geologischpedologische Vorgänge zur Entstehung einer meist nur wenige dm (3-6) mächtigen Deckschicht (Schönhals 1957a) geführt haben. Sie wird u. a. auch als Deckschutt, Decksediment (Semmel 1964, Plass 1966) und periglaziäre Deckzone (Kopp 1965) bezeichnet; kürzlich schlug Semmel (1973) den Begriff „Umlagerungszone“ vor.

Das unter periglazialen Klimabedingungen gebildete Substrat ist in den Mittelgebirgen und Beckenlandschaften flächenhaft verbreitet. Aber auch im norddeutschen Vereisungsgebiet sind schon in den 50er Jahren (Lемвке 1954; Liedtke 1958) und Mitte der 60er Jahre (Kopp 1965) sowie neuerdings von Kliewe \& Schultz (1970) Erscheinungen beschrieben worden, die sehr wahrscheinlich auf geopedologische Prozesse während der Jüngeren Tundrenzeit zurückzuführen sind. Zu dem gleichen Ergebnis führten auch Untersuchungen im Gebiet der Würmmoränen und -schotterterrassen des nördlichen Alpenvorlands (Jerz 1970, Semmel 1973).

\section{Charakteristische Eigenschaften der jungtundrenzeitlichen Deckschicht}

Die Deckschicht hat je nach den geologischen und orographischen Verhältnissen eine sehr unterschiedliche Körnung und petrographische Zusammensetzung. Kennzeichnend ist jedoch der immer vorhandene Schluffgehalt, wobei Grob- und Mittelschluff, also die typischen Fraktionen äolisch entstandener periglazialer Sedimente (z. B. Löß und Sandlöß) überwiegen. Der Schluffgehalt erreicht in Mittelgebirgslagen 40-60\%; in Lößgebieten ist der Schluffgehalt noch höher.

*) Anschriften der Verfasser: Prof. Dr. E. S c hönhals, Institut für Bodenkunde und Bodenerhaltung der Justus-Liebig-Universität Gießen, Ludwigstraße 23, 6300 Lahn-Gießen; Dr. Th. $\mathrm{J}$. P o e ts ch, Institut für Geographie und Wirtschaftsgeographie der Universität, Bundesstr. 55, 2000 Hamburg 13. 
Auf den hohen Anteil der äolisch bedingten Komponente in der jungtundrenzeitlichen Deckschicht hessischer Mittelgebirge wurde von Scнönhals (1957a) hingewiesen und deren boden- und standortskundliche Bedeutung hervorgehoben (SchöNHALs 1972, 1973, 1974). Mit der petrographischen Zusammensetzung der jungen Deckschicht und der Herkunft des Schluffs hat sich u. a. auch Ровтsch (1975) eingehend beschäftigt.

\section{Die Deckschicht in den Nördlichen Kalkalpen (Seefeld-Leutasch)}

Die bisherigen Untersuchungen über die Entstehung der Deckschicht haben ergeben, daß neben frostdynamischen Prozessen, vor allem Solifluktion und Kryoturbation i.w.S., äolische Vorgänge in einem \pm großen Umfang beteiligt waren, und zwar nicht nur in den einleitend erwähnten Gebieten, sondern auch in den Tiroler Kalkalpen (Schönhals $1957 b)$. So wurde in der Umgebung von Seefeld und Leutasch beobachtet, daß auf carbonatischen Fest- und Lockergesteinen eine braune Deckschicht liegt, die meist 3-6 dm erreicht, aber auch bis zu einem Meter mächtig werden kann. Sie wurde nach Ansicht von SснӧNHALs (1957b) während der Jüngeren Tundrenzeit auf einer durch frostdynamische Vorgänge entstandenen, kleinwellig-buckeligen Oberfläche (Primärrelief) in unterschiedlicher Mächtigkeit abgelagert, in den Kleindellen mächtiger als auf den Buckeln, wo die Deckschicht auch häufig fehlt. Dadurch war eine wichtige Voraussetzung für die Entstehung und den kleinräumigen Wechsel von Rendzinen, Braunerden und Podsolen gegeben.

Diese Ergebnisse werden von Zech \& Wölfel (1974) und Zech \& Neuwinger (1974) abgelehnt und erneut die schon von anderen Autoren geäußerte Auffassung vertreten, daß das braune Substrat durch chemische Verwitterung in situ entstanden sei. Die Hauptursache der Carbonatauswaschung ist nach Zech \& Neuwinger (1974) „die Akkumulation mächtiger, stark saurer ( $\mathrm{pH}$ des $\mathrm{O}_{\mathrm{F}}$ in $\mathrm{KCl}$ um 2,8) organischer Auflagen, welche die Carbonatlösung entlang bevorzugter Sickerwasserbahnen in hohem Maße beschleunigen".

\section{Neue Untersuchungen}

Die zuletzt zitierten beiden Arbeiten waren der Anlaß für neue genauere Untersuchungen im Gebiet von Seefeld und Leutasch; die Ergebnisse werden im folgenden mitgeteilt: Die Proben wurden in der früher schon untersuchten, jetzt aufgelassenen Schottergrube an der Straße Seefeld-Leutasch entnommen (Abb.1), und zwar aus dem unverwitterten Schottermaterial an der Profilbasis und dem schluffigen Sand im Hangenden. Das erwähnte Schottermaterial besteht zu 69,4\% (Gew. $\%$ ) aus Skelett $(\varnothing>2 \mathrm{~mm})$ und zu $30,6 \%$ aus Feinerde (Abb. 2a). Skelett und Feinerde wurden getrennt und unter zeitweiliger Erwärmung auf ca. $60^{\circ}$ so lange mit etwa $10 \%$ iger $\mathrm{HCl}$ behandelt, bis alle Carbonate zerstört waren ${ }^{1}$ ). Vom Skelettanteil waren 91,3\% und von der Feinerde 83,1 \% in verd. $\mathrm{HCl}$ löslich (Abb. 2a). In der Gesamtprobe des Kalkschotters (Feinerde und Skelett) sind demnach $88,8 \%$ in verd. $\mathrm{HCl}$ löslich. Es kann angenommen werden, daß dieser Anteil an Carbonaten auch während einer entsprechend langen Zeit gelöst wird. Es würden dann 11,2\% unlösliche Bestandteile zurückbleiben, vorwiegend Quarz und Silikate.

Legt man diese beiden Kennwerte zugrunde, dann kann man die Substratvolumina berechnen, die zur Entstehung einer Volumeneinheit Boden erforderlich wären. Hierbei muß jedoch das unterschiedliche Porenvolumen des Schotters und des Bodens berücksichtigt werden. Unter der Annahme, daß das Porenvolumen des Schotters 40\% und des

1) Hierbei können auch nichtcarbonatische Bestandteile, vor allem die feinsten Körner, angegriffen worden sein; manche Komponenten, z. B. Apatit und Eisenoxide, wurden vielleicht sogar vollständig gelöst. Durch diese möglichen Vorgänge werden jedoch die dargestellten Ergebnisse nur unwesentlich beeinflußt. 


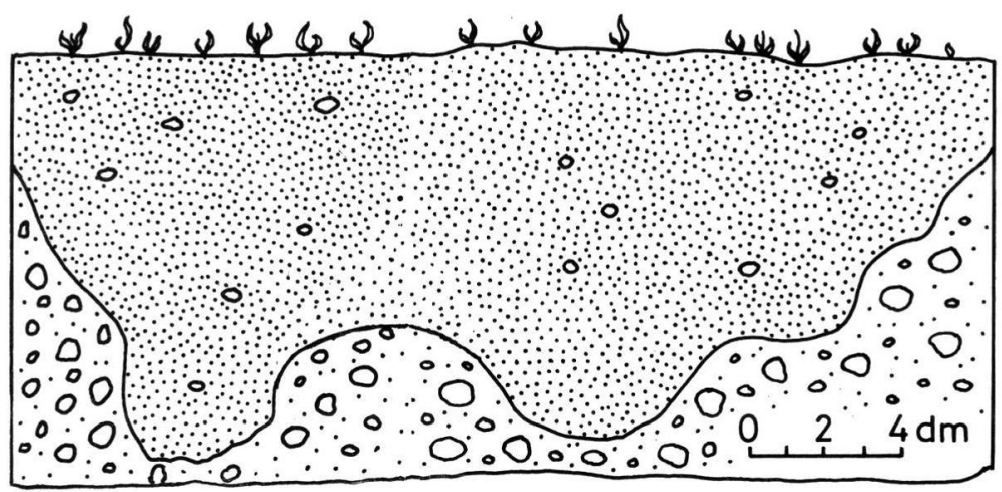

Abb. 1: Die Überlagerung des Kalkschotters durch braunen schluffigen Sand in der aufgelassenen Kiesgrube an der Straße von Seefeld nach Leutasch.

Bodens $60 \%$ beträgt, muß der Wert für den unlöslichen Rückstand des Schotters, also 11,2\%, mit dem Faktor 1,5 (6:4) multipliziert werden. Für 16,8 Volumeneinheiten Boden wären demnach 100 Volumeneinheiten des Ausgangsgesteins notwendig. Nimmt man an, daß $1 \mathrm{~m}^{3}$ Boden 16,8\% des Volumens des Ausgangsgesteins darstellt, dann würde dieses Volumen $5,95 \mathrm{~m}^{3}$ betragen. Für die Entstehung eines carbonatfreien Solums von $1 \mathrm{~m}$ Mächtigkeit müßten also Kalkschotter in einer Mächtigkeit von 5,95 m entcarbonatisiert werden. Auch wenn man von einem geringmächtigeren Solum ausgeht, erscheint in Anbetracht der Profilmorphologie und der Geländesituation eine Entcarbonatisierung in situ von solchen Gesteinsmassen als sehr unwahrscheinlich.

Weitere Anhaltspunkte über das Vorkommen einer Deckschicht sind aus dem Vergleich der Körnung der carbonatfreien Feinerde des Kalkschotters und des Solums zu gewinnen (Tab. 1). Die Bestimmung der Körnung erfolgt nach Schlichting \& Blume (1966).

Tab. 1: Korngrößenverteilung in der carbonatfreien Feinerde des Schotters und des Solums

Fraktion

Korndurchmesser in $\mu \mathrm{m} \quad$ Feinerde des Schotters $\quad$ Feinerde des Solums

\begin{tabular}{lccr}
\hline Grobsand & $630-2000$ & $32,4 \%$ & $11,0 \%$ \\
Mittelsand & $200-630$ & $18,8 \%$ & $15,5 \%$ \\
Feinsand & $63-200$ & $17,4 \%$ & $26,3 \%$ \\
Grobschluff & $20-63$ & $14,7 \%$ & $20,4 \%$ \\
Mittelschluff & $6,3-20$ & $10,9 \%$ & $20,2 \%$ \\
Feinschluff & $2-6,3$ & $4,3 \%$ & $3,0 \%$ \\
Ton & $<2$ & $1,5 \%$ & $3,6 \%$
\end{tabular}
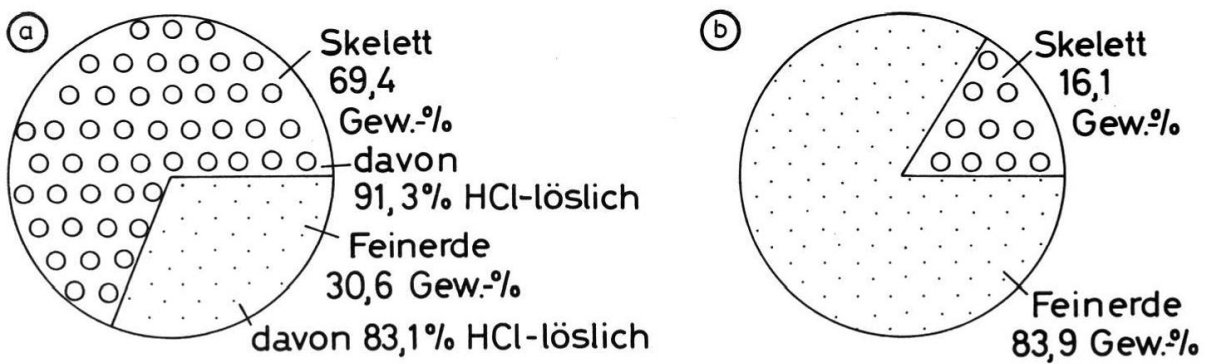

Abb. 2: Der Skelett- und Feinerdeanteil des Kalkschotters (a) und des Solums (b). 
Wie aus den Kennwerten der Tab. 1 und den Abb. 3 a u. b zu ersehen ist, bestehen in der Korngrößenverteilung der carbonatfreien Feinerde des Kalkschotters und der des Feinmaterials in seinem Hangenden wesentliche Unterschiede: Die Feinerde des Kalkschotters hat ein Maximum im Grobsand, die des Solums im Feinsand; im Kalkschotter ist der Schluffgehalt gering, im Boden dagegen sehr hoch. Hervorzuheben ist noch, daß im Solum der Mittel- und Grobschluff nach dem Feinsand die Hauptanteile ausmachen. Die Körnung dieses Materials hat damit eine große Ähnlichkeit mit der des Sandlösses.

Zusätzlich ist aufschlußreich zu untersuchen, welche Körnung die nichtcarbonatischen, d. h. HCl-unlöslichen Bestandteile des Skeletts im Schotter aufweisen. Vor allem das carbonatische Skelett könnte doch durch seinen starken Anteil im Schotter der potentielle Lieferant der Feinerde im Solum sein. Wenn man allerdings, wie oben dargestellt, diesen Skelettanteil mit $\mathrm{HCl}$ behandelt, besteht der unlösliche Rückstand wieder zu $92 \%$ aus Skelettbestandteilen, der Feinerdeanteil macht also nur $8 \%$ aus.
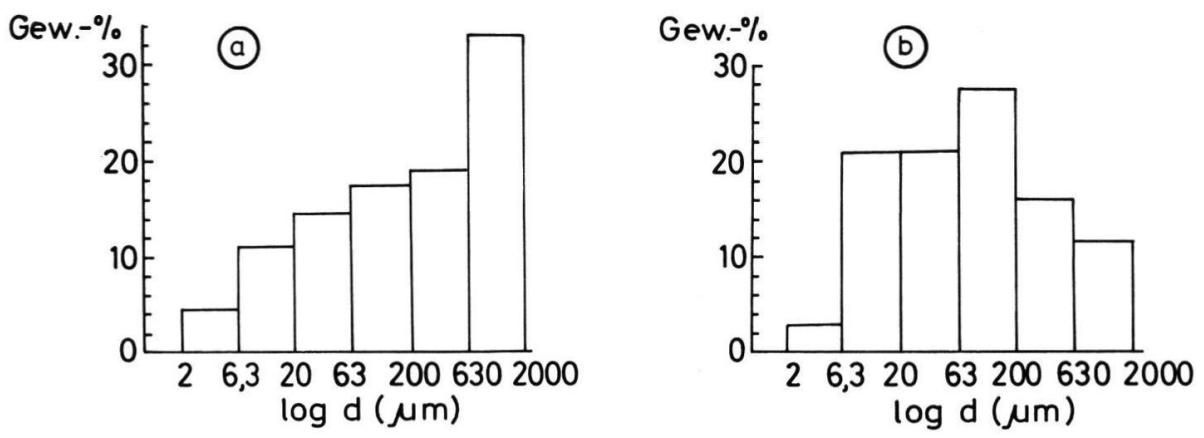

Abb. 3: Die Korngrößenverteilung in der carbonatfreien Feinerde des Schotters (a) und des Solums (b).

Tab. 2: Körnung des carbonatfreien Lösungsrückstandes nach der HCl-Behandlung der Skelettfraktion des Schotters

Fraktion

Korndurchmesser in $\mu \mathrm{m} \quad$ Feinerde des Lösungsrückstandes

Grobsand

Mittelsand

Feinsand

Schluff und Ton

$$
\begin{gathered}
630-2000 \\
200-630 \\
63-200 \\
<63
\end{gathered}
$$

$48,1 \%$
$39,8 \%$
$11,0 \%$
$1,1 \%$

$48,1 \%$

$1,1 \%$

Sowohl der hohe Anteil der Partikel von $\phi>2 \mathrm{~mm}$ im Lösungsrückstand als auch die Körnung seines Feinerde-Anteiles (Tab. 2) sprechen gegen die Annahme, daß das Solum aus dem Schotter hervorgegangen ist.

Außer der Körnung der carbonatfreien Feinerde des schluffig-sandigen Schotters und des Bodens wurde für die Untersuchung auch die Schwermineralanalyse herangezogen. Die unmittelbare Messung anhand von Mikroaufnahmen erfaßte Korngrößen von 36$360 \mu \mathrm{m}$. Gemessen wurde dabei der sogenannte größte Durchmesser lp des jeweiligen Korns, d.h. die Entfernung seiner zwei am weitesten voneinander entfernten Punkte (Hörnsten 1959, zitiert in Müller 1964). Die Ergebnisse sind in den Abb. 4 a und 4 b dargestellt. Sie lassen die deutlichen Unterschiede erkennen, die zwischen der Körnung der Schwerminerale des Schotters und des Solums bestehen: In beiden Fällen zeigt zwar die Kornverteilung ein Maximum im Bereich zwischen 110-150 $\mu \mathrm{m}$, die Fraktion 63$110 \mu \mathrm{m}$ ist aber im Solum ungleich stärker vertreten $(47,5$ Korn-0/0) als im Schotter 

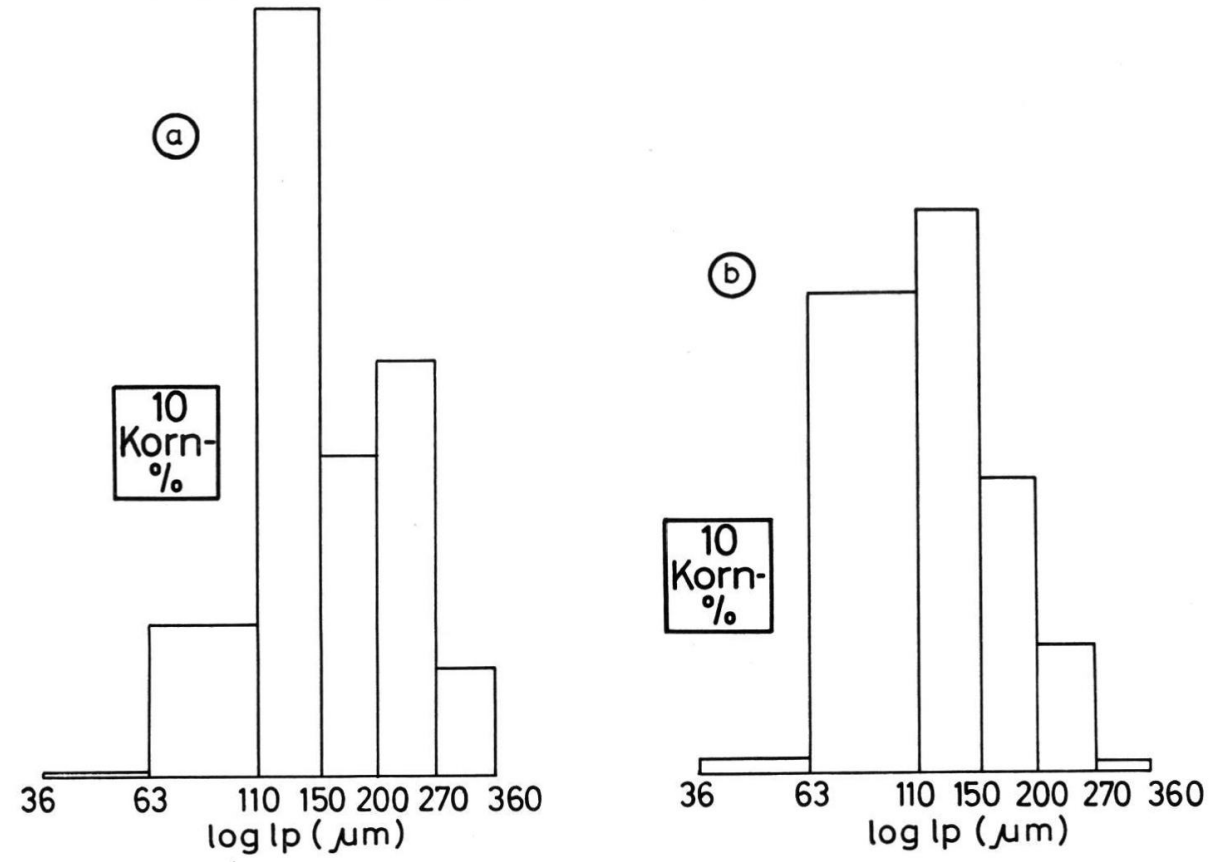

Abb. 4: Die Körnung der Schwerminerale in der Feinerde des Schotters (a) und des Solums b).

$(15,0$ Korn-0\%). Dabei ist hervorzuheben, daß nur ein Teil der Schwerminerale in den feineren Fraktionen auf mögliche pedogenetische Verwitterung und damit Zerkleinerung größerer Mineralindividuen an Ort und Stelle zurückgeführt werden kann. Viele Schwerminerale zeigen auch noch in den feineren Fraktionen ihre ursprüngliche Kristallform (Taf. I). Dies trifft vor allem für viele Zirkone, Rutile, Disthene und einige Granate zu.

Einen weiteren Hinweis auf schwache Verwitterung liefert auch die Feststellung, daß der Granat-Gehalt im Solum deutlich höher ist als im Kalkschotter (Tab. 3). Granat ist bekanntlich ein gegen die Verwitterung wenig stabiles Schwermineral; der relativ hohe Gehalt an frischen Granaten im Substrat muß daher andere Ursachen haben.

Tafel I

Schwerminerale aus der Feinerde der Deckschicht (Solum) und des Kalkschotters.

Deckschicht: Fig. 1: Zirkon; Fig. 2: Rutil; Fig. 3: Disthen; Fig. 4: Granat.

Kalkschotter: Fig. 5: Staurolith; Fig. 6: Disthen; Fig. 7-10: Granat.

Zirkon und Rutil aus der Deckschicht (Fig. 1 u. 2) weisen noch pyramidale und prismatische Kristallflächen auf. Der Disthen (Fig. 3) ist wegen seiner ausgeprägten Spaltbarkeit (3 Spaltsysteme) bei der Umlagerung möglicherweise mechanisch zerkleinert worden. Beim Granat aus der Deckschicht (Fig. 4) und dem Kalkschotter, bes. Fig. 8 und 10, sind die Kristallflächen (110 ?) noch deutlich zu erkennen. Für den Disthen aus dem Kalkschotter (Fig. 6) gilt das gleiche wie für den Disthen aus der Deckschicht (Fig. 3). Abbildungsmaßstab: Fig. 1, 2 u. $4=250: 1$; Fig. $3=200: 1$; Fig. $5-10=160: 1$. 

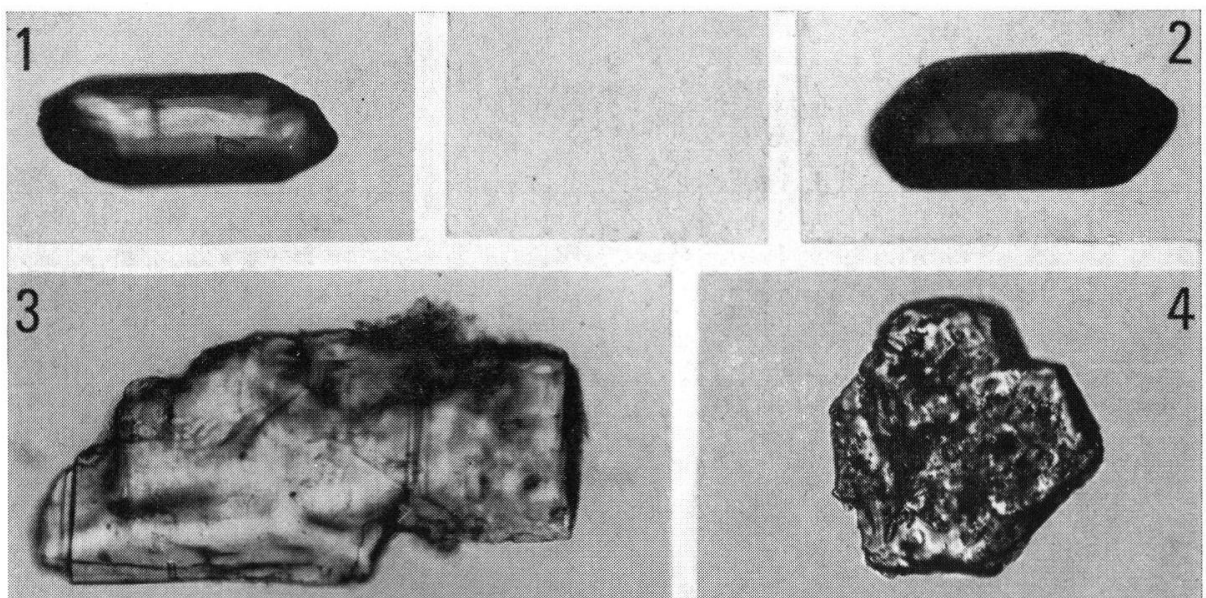

4

5
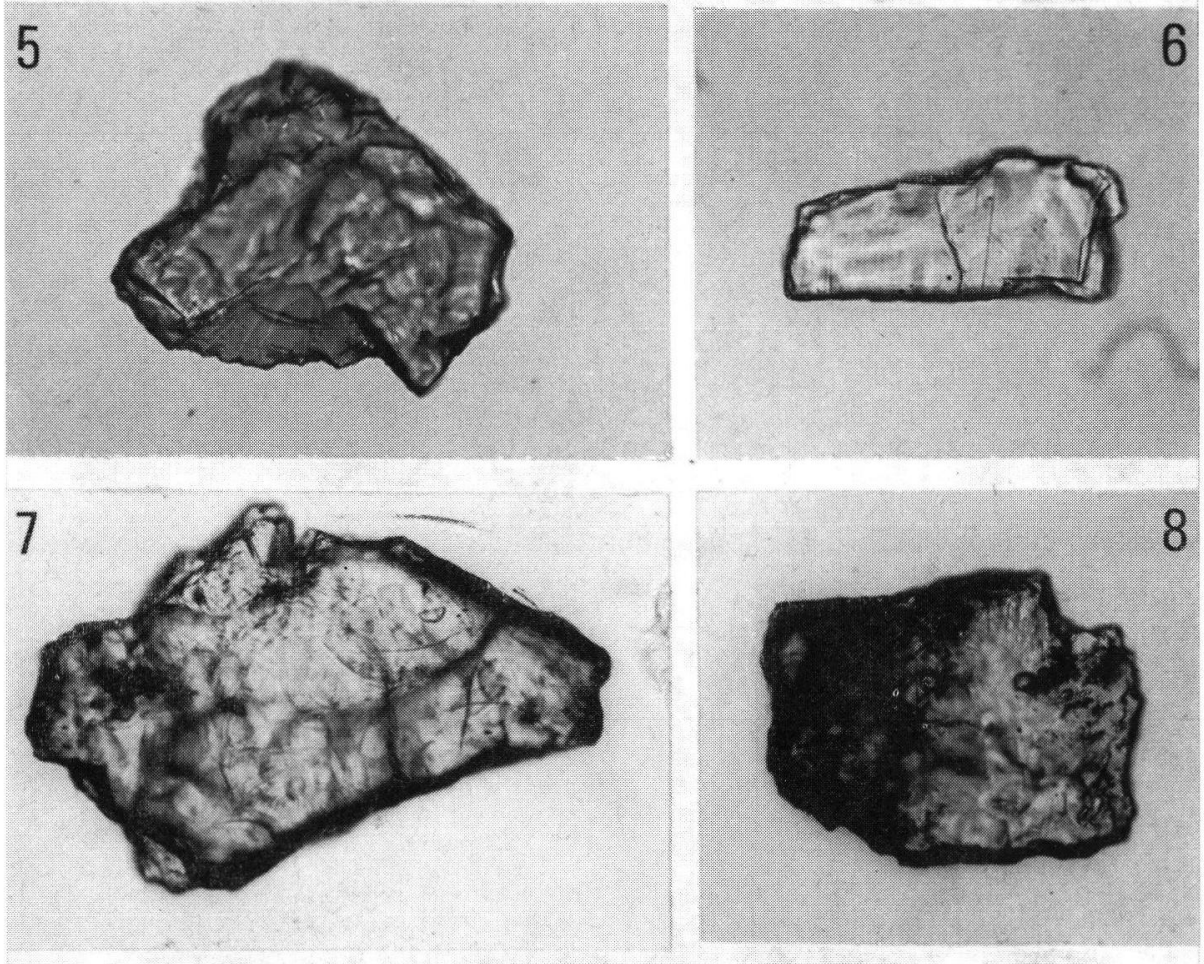

9
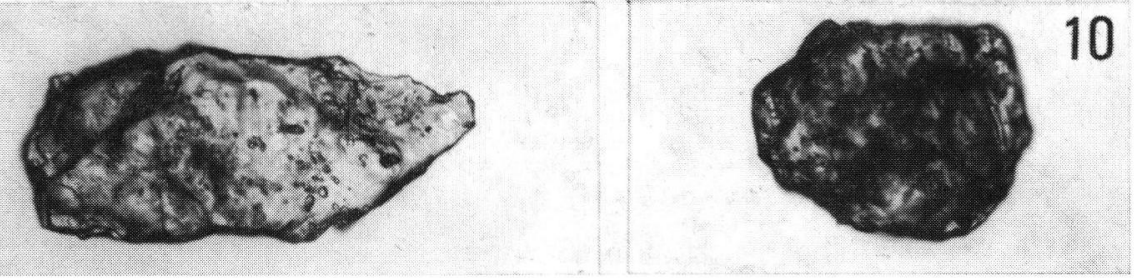
Tab. 3: Mineralogische Zusammensetzung der Schwerminerale im Korngrößenbereich 36-200 $\mu \mathrm{m}$. Summe der transparenten Schwerminerale 100\%. Anteil der opaken Körner ist bezogen auf die gesamten Schwerminerale. Angaben jeweils in Korn- 0 a.

$$
\begin{array}{cc}
\text { Boden } & \text { Kalkschotter } \\
(\mathrm{n}=600) & (\mathrm{n}=553)
\end{array}
$$

\begin{tabular}{lrcc}
\hline Opak-Minerale & \multicolumn{2}{c}{23,3} & \multicolumn{2}{c}{18,1} \\
Granat & 50,2 & 34,2 \\
Zirkon & 0,4 & 2,2 \\
Rutil & 2,6 & 0,4 \\
Turmalin & 0,4 & 7,9 \\
Epidot-Reihe & 12,4 & 0,4 \\
Titanit & 0,2 & 0,4 \\
Pyroxen & - & 17,8 \\
Grüne gemeine Hornblende & 12,2 & 9,9 \\
Staurolith & 10,7 & 2,6 \\
Disthen & 2,3 & 0,2 \\
Sillimanit & - & 20,0 \\
Aggregate & 4,6 & 3,5 \\
Unbekannt & 4,0 & 99,5 \\
\hline
\end{tabular}

\section{Diskussion der Ergebnisse}

Für das Vorhandensein einer Deckschicht im Untersuchungsgebiet und gegen die Möglichkeit einer Feinmaterial-Anhäufung in situ als Residuum einer Schotter-Entcarbonatisierung sprechen mehrere Gründe:

a) Für die zu beobachtenden Solum-Mächtigkeiten müßten sehr große Mengen von Carbonat-Schottern als Ausgangsgestein aufgelöst worden sein. Wäre dieses Solum in der Tat hauptsächlich ein Verwitterungsresiduum der Kalkschotter, hätte sich bei der so vorausgesetzten intensiven chemischen Verwitterung aus den Silikaten auch wesentlich mehr Ton bilden müssen als die Deckschicht enthält.

b) Nach der Behandlung des Schottermaterials mit verdünnter Salzsäure zeigt das Residuum eine stark einseitige Korngrößenverteilung mit ausgeprägtem Maximum im Grobsandbereich. Im Gegensatz dazu verschiebt sich die Korngrößenverteilung im Boden zugunsten der feineren Fraktionen mit einem Modus im Feinsand / Grobschluff-Bereich.

c) Die Korngrößenverteilung innerhalb der Schwermineralfraktion zeigt deutliche Unterschiede zwischen der Deckschicht und dem Schotter; die Deckschicht enthält viel mehr kleinere Schwerminerale als ihr Untergrund.

d) Dieser Effekt ist jedoch nicht dominant auf die pedogenetisch bedingte ortsständige Schwermineral-Zerkleinerung (Verwitterung) zurückzuführen. Vielmehr sind die feinkörnigen Schwerminerale in der Deckschicht als Anzeiger und Bestandteile eines von vornherein feinkörnigeren Sediments anzusehen. Das beweisen mehrere idiomorphe Kristalle und gleichzeitig die Tatsache, daß die als wenig stabil geltenden Granate in der Deckschicht (Solum) viel stärker vertreten sind als im Schotter. Außerdem weist die Körnung der Deckschicht und ihrer Schwermineralfraktion auf eine äolische Genese hin. Dies geht vor allem aus dem hohen Gehalt an Grobschluff und Feinsand sowie aus dem höheren Anteil feinerer Granate und anderer Schwerminerale hervor. 
Aufgrund dieser Ergebnisse und nach Beobachtungen an zahlreichen Profilen kommt für die Bildung der Deckschicht ein äolischer Materialtransport als dominanter Vorgang in Betracht. Dabei handelte es sich u. E. nicht um die Anwehung von Feinmaterial aus größerer Entfernung, sondern um kleinräumigen Transport, wie dies auch von anderen Sedimenten der Jüngeren Tundrenzeit bekannt ist. Es überrascht daher nicht, daß zwischen Deckschicht und Kalkschotter eine mineralogische Verwandtschaft zu erkennen ist.

Um keine Mißverständnisse aufkommen zu lassen, sei noch erwähnt, daß unter den jeweils herrschenden Klima- und Vegetationsbedingungen selbstverständlich auch die chemische Verwitterung wirksam war und ist. So beobachtet man an der Grenze Deckschicht/Schotter angewitterte carbonatische Skelettbestandteile. Im übrigen ist die Grenze zwischen Deckschicht bzw. Boden und Untergrund sehr scharf ausgebildet; ein Übergangshorizont $\mathrm{B}_{\mathrm{V}} \mathrm{C}_{\mathrm{v}}$ oder $\mathrm{C}_{\mathrm{v}}$ fehlt fast immer. Auch dies ist ein Hinweis auf eine relativ geringe chemische Verwitterung. Schließlich sei noch erwähnt, daß das $\mathrm{pH}(\mathrm{KCl})$ im $\mathrm{B}_{\mathrm{v}}$-Horizont von etwa $3 \mathrm{dm}$ mächtigen Braunerden Werte um 6 erreicht, was auch aus den Untersuchungen von Zech \& Neuwinger (1974) hervorgeht. Die von Zech \& Neuwinger als Ursache der starken Carbonatlösung genannten sauren Humusauflagen haben demnach schon in einer Tiefe von wenigen $\mathrm{dm}$ nur noch einen schwachen Einfluß, der bei mächtigeren Deckschichten i. a. noch weiter abnimmt. Wo die Deckschicht in primär angelegten tieferen Kleindellen eine größere Mächtigkeit erreichte, waren die ökologischen Voraussetzungen für eine erhöhte Produktion und Anreicherung organischer Stoffe und damit für die Podsolierung gegeben (vgl. SснӧNHALs 1957; Abb. 6 u. 7). An feuchteren Schatthängen sind unter moos- und vacciniumreichen Fichtenmischwäldern die Bedingungen für die Entstehung von Rohhumusauflagen und damit für die Podsolierung besonders günstig. An solchen Stellen ist dann auch ein $\mathrm{C}_{\mathrm{v}}$-Horizont ausgebildet, aber fast immer nur unter den primär angelegten tieferen Kleindellen (vgl. auch ZECH \& NEUwINGER 1974: Abb. 2).

Die Untersuchungen erbrachten den Nachweis, daß in der Umgebung von Seefeld und Leutasch über carbonatischen Gesteinen eine Deckschicht liegt, die nicht durch chemische Verwitterung, sondern unter periglazialen Klimabedingungen entstanden ist. Sie besteht überwiegend aus Schluff sowie Fein- und Mittelsand. Bei der Bildung der Deckschicht waren äolische Umlagerungen dominant. Nach den vorliegenden Ergebnisseen über Untersuchungen an spätglazialen Deckschichten kann angenommen werden, daß auch das aus Tirol beschriebene Substrat während der Jüngeren Tundrenzeit gebildet worden ist.

\section{Schriftenverzeichnis}

Hörnsten, A. (1959): A method an a set of apparatus for mineralogic - granulometric analysis with a microscope. - Bull. Geol. Inst. Univ. Uppsala, 38: 105-137; Uppsala.

KLIEwE, H. \& SchulTz, H.-J. (1970): Die periglaziäre Fazies im Jungmoränengebiet nördlich der pommerschen Eisrandlage. - Pet. geogr. Mitt., Erg. H., 274: 255-263; Gotha.

Kopp, D. (1965): Die periglaziäre Deckzone (Geschiebedecksand) im nordostdeutschen Tiefland und ihre bodenkundliche Bedeutung. - Ber. geol. Ges. DDR, 10: 739-771; Berlin.

Lembкe, H. (1954): Die Periglazialerscheinungen im Jungmoränengebiet westlich des Oderbruchs bei Freienwalde. - Gött. geogr. Abh., 16: 55-95; Göttingen.

LiEDTKE, H. (1958): Frostbodenstrukturen aus dem norddeutschen Jungmoränengebiet. - Wiss. Z. Univ. Berlin, math. nat. R., 7: 359-376; Berlin.

MüLLER, G. (1964): Methoden der Sediment-Untersuchung. - Stuttgart (Schweizerbart).

Plass, W. (1966): Braunerden und Parabraunerden in Nordhessen. - Z. Pflanzenernähr. Düng., Bodenkde., 114, 1 : 12-26; Weinheim.

Poetsch, T. J. (1975): Untersuchungen von bodenbildenden Deckschichten unter besonderer Berücksichtigung ihrer vulkanischen Komponente. - Gießener geol. Schr., 4: 180 S., 29 Abb., Tab. Anhang; Gießen (Lenz). 
Schlichting, E. \& Blume, H.-P. (1966): Bodenkundliches Praktikum. - Hamburg u. Berlin (Parey).

SCHÖNHALS, E. (1957a): Spätglaziale äolische Ablagerungen in einigen Mittelgebirgen Hessens. Eiszeitalter u. Gegenwart, 8: 5-17; Öhringen (Rau).

- (1957b): Späteiszeitliche Wind-Ablagerungen in den Nördlichen Kalkalpen und die Entstehung der Buckelwiesen. - Natur und Volk, 87: 317-328; Frankfurt a. M.

- (1972): Die bodenkundliche und standortskundliche Bedeutung der geologischen Vorgänge während des Spätglazials. - Vortr.-Tag. d. Arbeitsgem. f. Forstl. Vegetationskde., 33-49; Bonn.

- (1973): Zur Landesnatur Mittelhessens. - Mitt. dt. bodenk. Ges., 17: 11-62; Göttingen.

- (1974): Die Bedeutung geologischer, insbesondere quartärgeologischer Vorgänge für die Bodenbildung in Hessen. - Mitt. dt. bodenk. Ges., 18: 11-17; Göttingen.

Semmel, A. (1964): Junge Schuttdecken in hessischen Mittelgebirgen. - Notizbl. hess. L.-Amt Bodenforsch., 92: 275-285; Wiesbaden.

- (1973): Periglaziale Umlagerungszonen auf Moränen und Schotterterrassen der letzten Eiszeit im deutschen Alpenvorland. - Z. Geomorph. N. F., Suppl. Bd., 17: 118-137; Berlin u. Stuttgart (Borntraeger).

ZECH, W. \& Neuwinger, I. (1974): Podsolbildung aus kalkreichen Substraten. Beobachtungen in den Tiroler Kalkalpen bei Seefeld. - Forstwiss. Centralbl., 93: 179-191; Hamburg u. Berlin (Parey).

- \& WöLfel, U.: Untersuchungen zur Genese der Buckelwiesen im Kloaschautal. - Forstwiss. Centralbl., 93: 137-155; Hamburg u. Berlin (Parey). 